Supporting Information

\title{
Expedient Synthesis of Antofine and Cryptopleurine via Intramolecular 1,3-Dipolar Cycloaddition
}

Sanghee Kim,* Yun Mi Lee, Jaekwang Lee, Taeho Lee, Ye Fu, Yanling Song, Jihee Cho, and Deukjoon Kim

\section{Contents}

General Experimental Method

S2

${ }^{1} \mathrm{H}$ NMR and ${ }^{13} \mathrm{C}$ NMR spectra of compound 6

S3-S4

${ }^{1} \mathrm{H}$ NMR spectrum of compound 7

S5

${ }^{1} \mathrm{H}$ NMR and ${ }^{13} \mathrm{C}$ NMR spectra of compound $\mathbf{8}$

S6-S7

${ }^{1} \mathrm{H}$ NMR and ${ }^{13} \mathrm{C}$ NMR spectra of compound 9

S8-S9

${ }^{1} \mathrm{H}$ NMR and ${ }^{13} \mathrm{C}$ NMR spectra of compound $\mathbf{1 3}$

S10-S11

${ }^{1} \mathrm{H}$ NMR and ${ }^{13} \mathrm{C}$ NMR spectra of compound $\mathbf{1 5}$

S12-S13

${ }^{1} \mathrm{H}$ NMR spectrum of compound $\mathbf{1 2}$ from $\mathbf{6}$

$\mathrm{S} 14$

${ }^{1} \mathrm{H}$ NMR and ${ }^{13} \mathrm{C}$ NMR spectra of compound 12 from 15

S15-S16

${ }^{1} \mathrm{H}$ NMR and ${ }^{13} \mathrm{C}$ NMR spectra of compound $\mathbf{1 6}$

S17-S18

${ }^{1} \mathrm{H}$ NMR and ${ }^{13} \mathrm{C}$ NMR spectra of compound $\mathbf{1 8}$

S19-S20

${ }^{1} \mathrm{H}$ NMR and ${ }^{13} \mathrm{C}$ NMR spectra of compound $\mathbf{1 9}$

S21-S22 
General. All chemicals were reagent grade and used as purchased. All reactions were performed under an inert atmosphere of dry argon or nitrogen using distilled dry solvents. Reactions were monitored through TLC analysis using silica gel 60 F-254 thin layer plates. Compounds were visualized on TLC plate under UV light and by spraying with $\mathrm{KMnO}_{4}$ or anisaldehyde solutions. Flash column chromatography was carried out on silica gel 60 (230-400 mesh). Melting points are uncorrected. ${ }^{1} \mathrm{H}$ NMR and ${ }^{13} \mathrm{C}$ NMR spectra were recorded in $\delta$ units relative to deuterated solvent as internal reference by 300, 500, or $600 \mathrm{MHz}$ NMR instrument. Mass spectra (MS) were recorded at 70 or 30 $\mathrm{eV}$ using electron impact (EI) or fast atom bombardment (FAB). High-resolution mass spectra (HRMS) were recorded using EI, FAB, or chemical ionization (CI). 

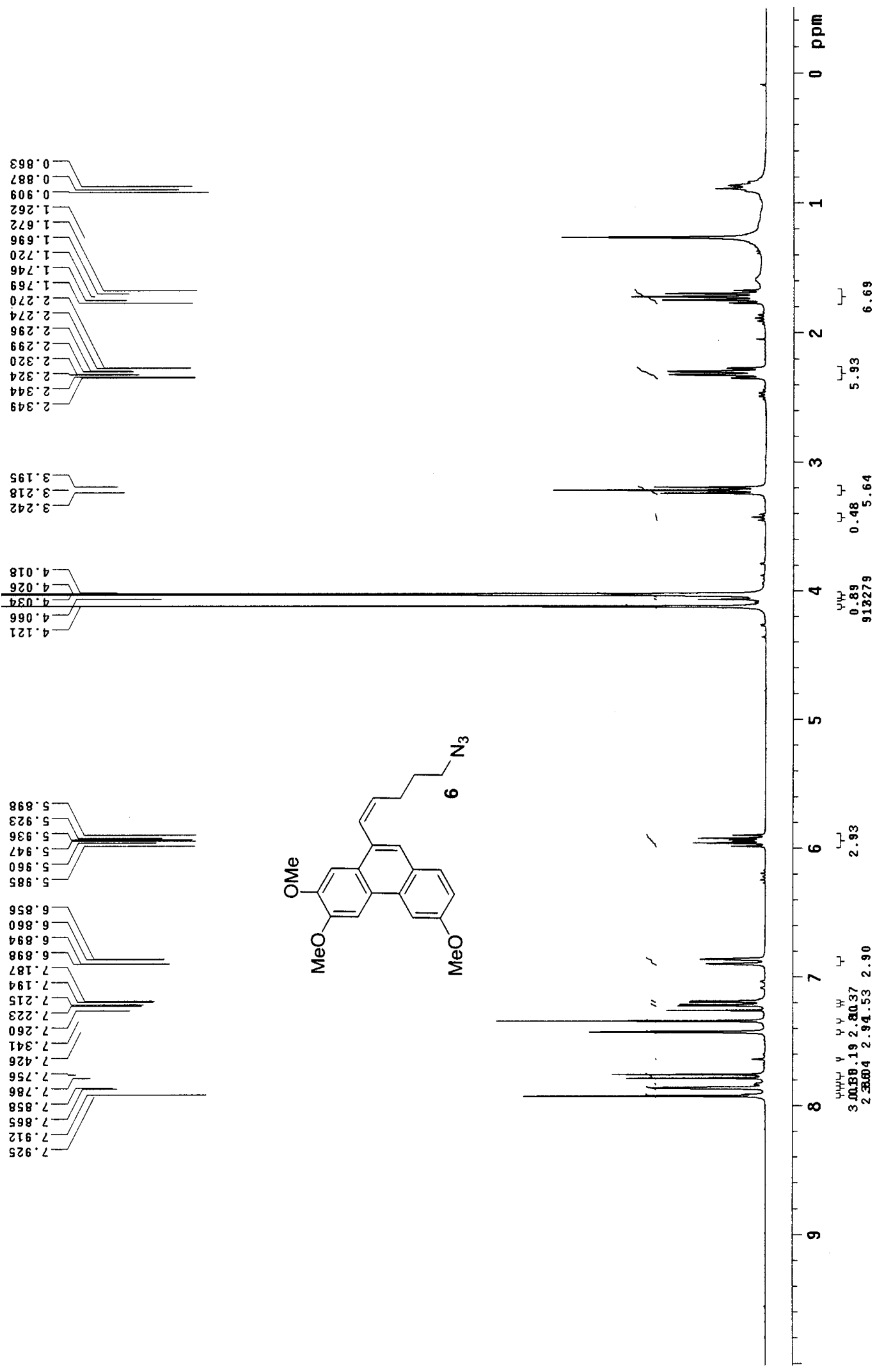

810.67

$920^{\circ} \rightarrow 1$

$+ 8 \varepsilon _ { 0 } ^ { \circ } \circ \longdiv { 1 2 }$

$990^{\circ} \circ$

โปโ

$868 \cdot 5$

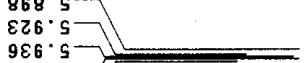

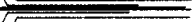

$096 \cdot \mathrm{s}$

$586 \cdot \mathrm{s}$

$958 \cdot 9$

$098^{\circ} \cdot$

$668^{\circ} \cdot$

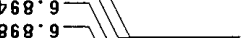

.

281.2

$\checkmark 6 \mathrm{~T} \cdot \mathrm{L}$

SI $2 \cdot 2 \square \underline{ }$

$\varepsilon z 2 \cdot<-$

$092 \cdot\llcorner$

It $\varepsilon^{\circ} \angle$

$926 \cdot 2$

$95<<$

$98<\cdot L-\sqrt{\sqrt{1}}$

$858^{\circ} \mathrm{L}$

$598^{\circ} \mathrm{L}$

II 6.

$526 \cdot 2$ 
$028.92 \square$
$008 \cdot 82$

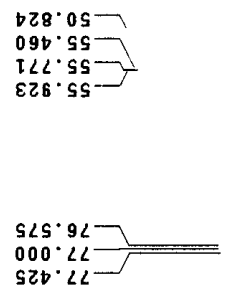

oIs. $\varepsilon 0 \tau$

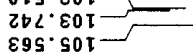

$600 \cdot 517$

$082^{\circ} \cdot 621$

9Z8.bZI

$6 I 9.52 I$
EOS.

999.82

$500.0 \varepsilon 1$

$89 t^{\circ} 0 \varepsilon \tau$

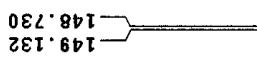

$100.8 \mathrm{st}$

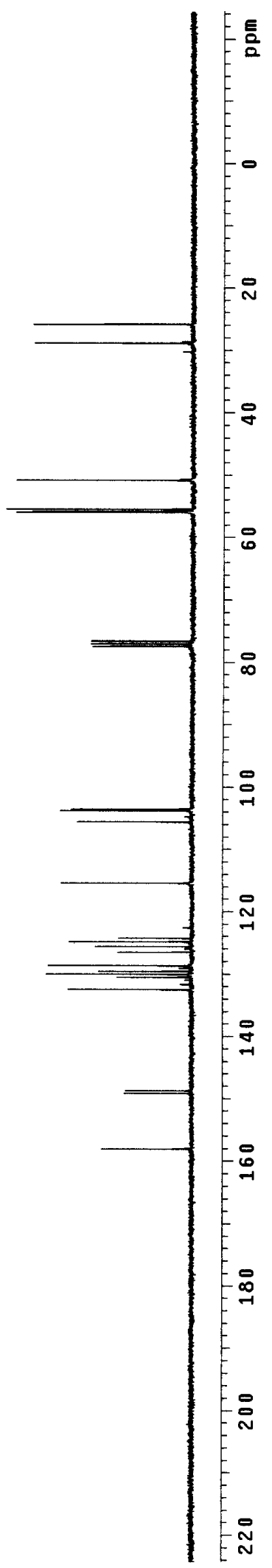



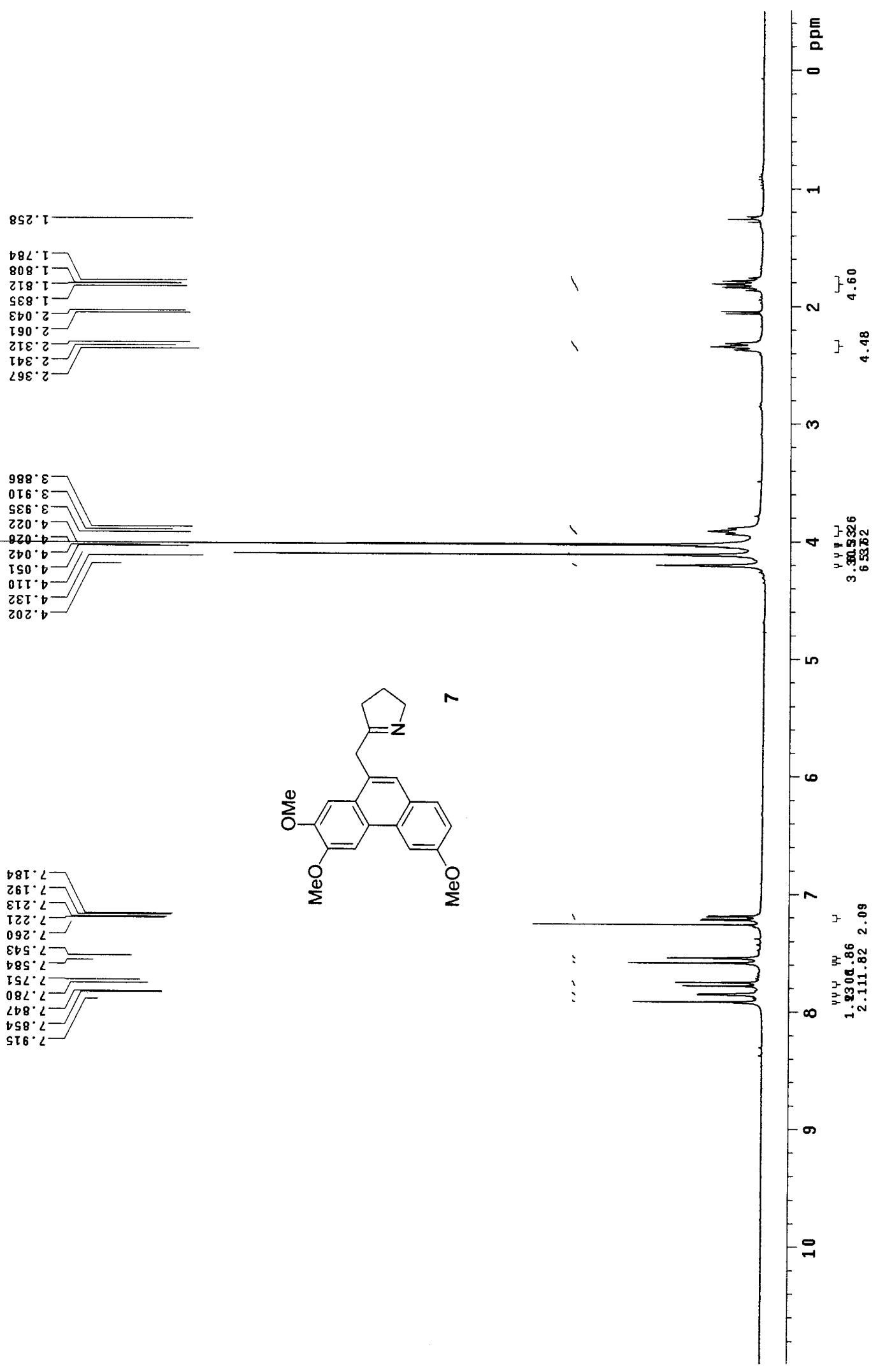


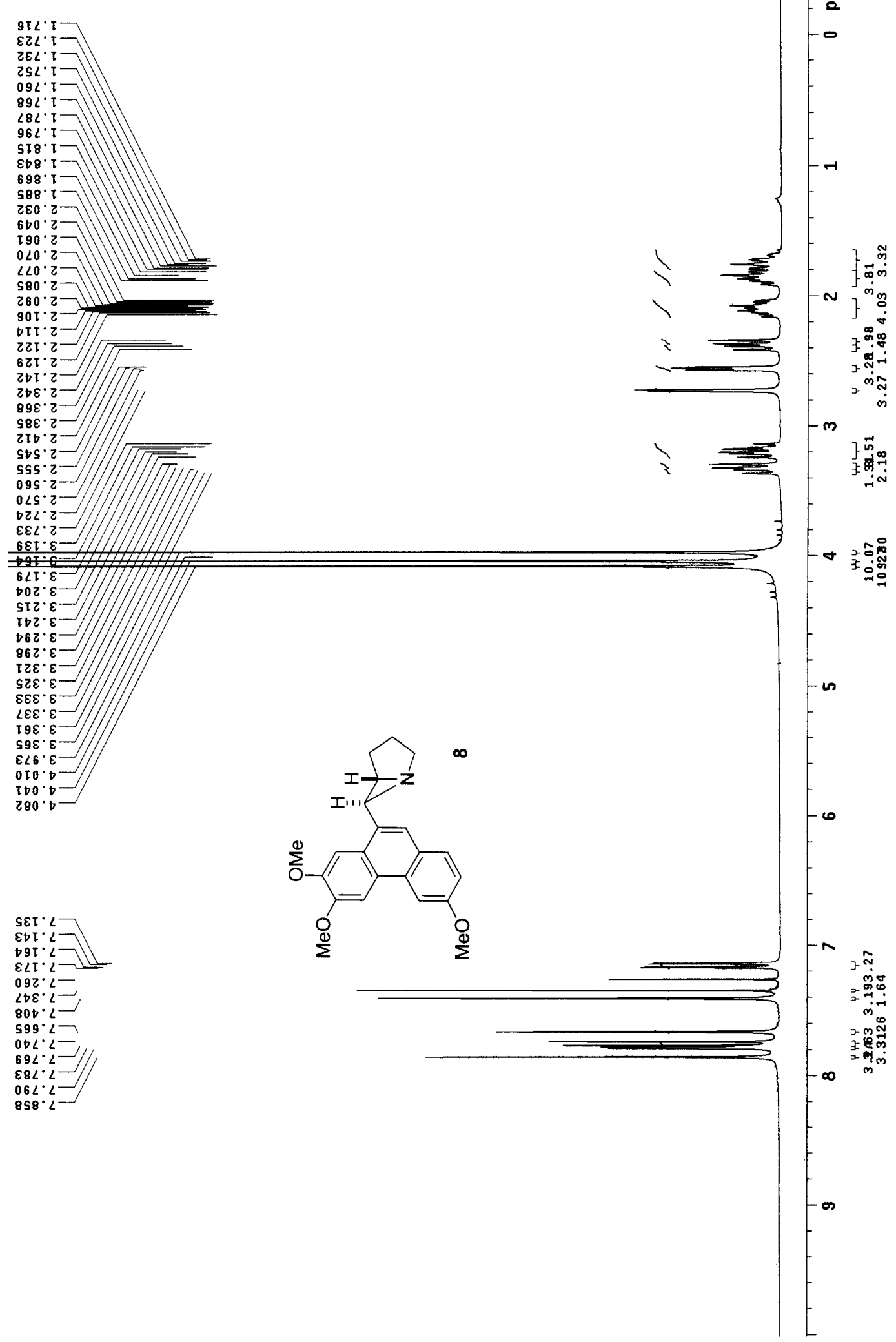




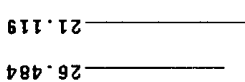

$6 \varepsilon \tau \cdot 8 \varepsilon$

$800 \cdot 86$

$66^{\circ} \mathrm{ZS}$
$8 \mathrm{SE} \cdot \mathrm{SS}$

$9 \varepsilon S . s S$

bZ8. SS

SLS.9L

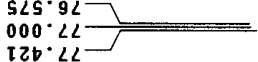

$\tau 2 b^{\circ} \angle L$

$829^{\circ} \varepsilon 0 \tau$

IS9.80I-

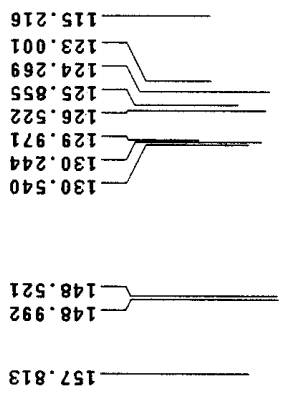

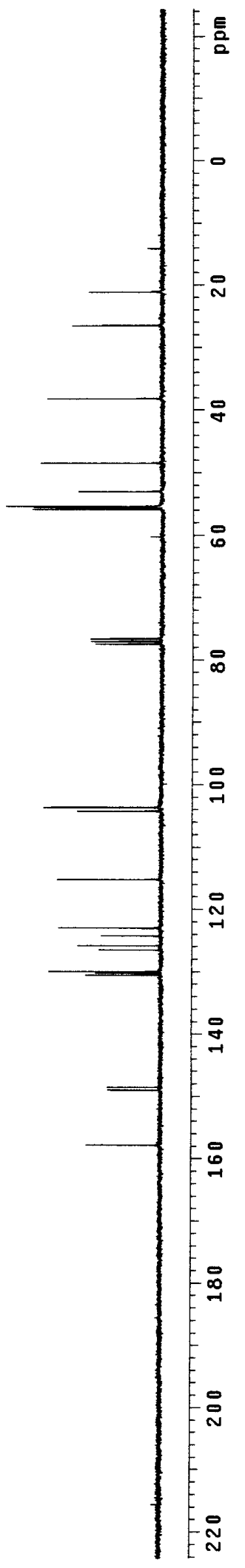




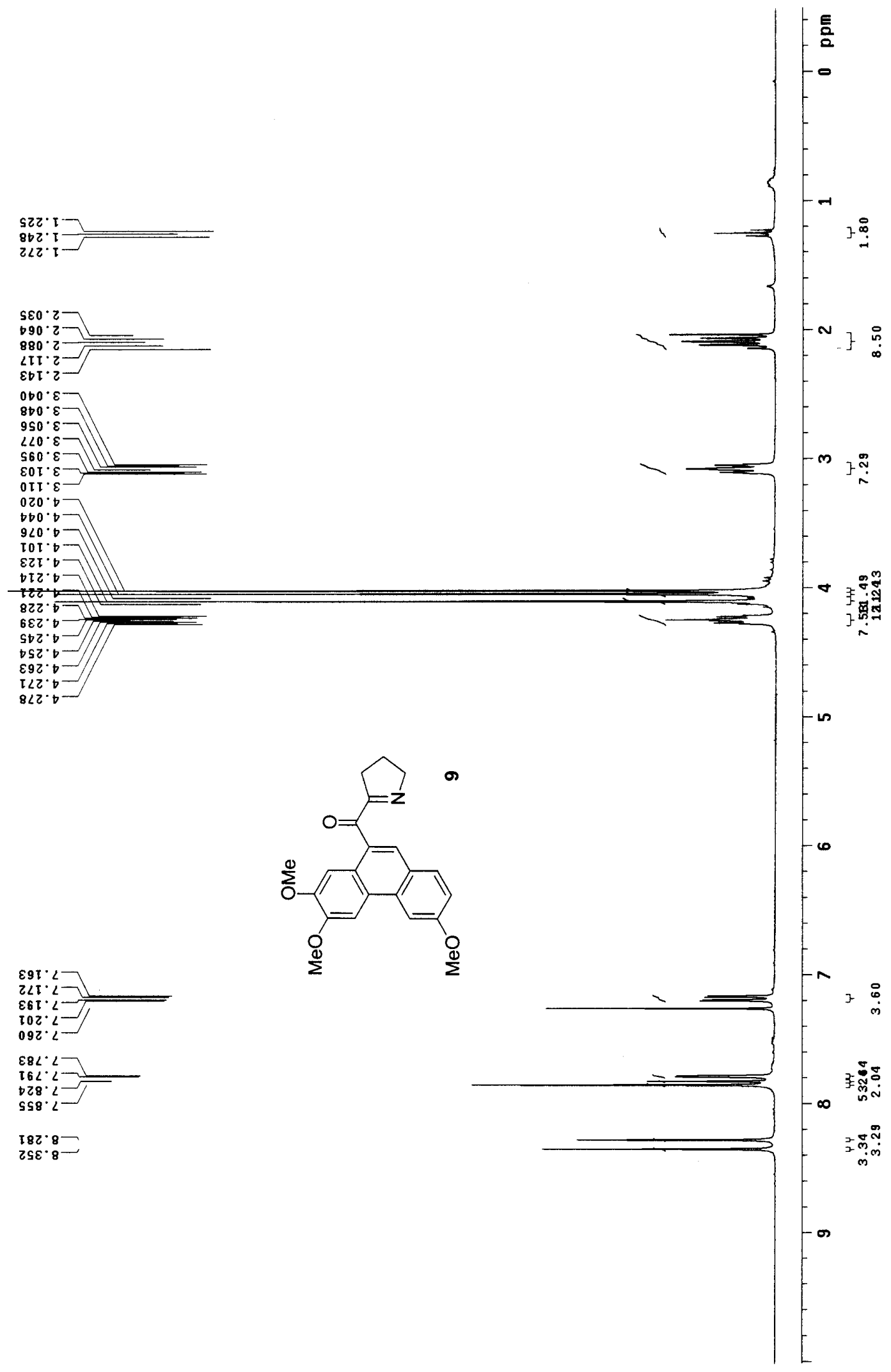




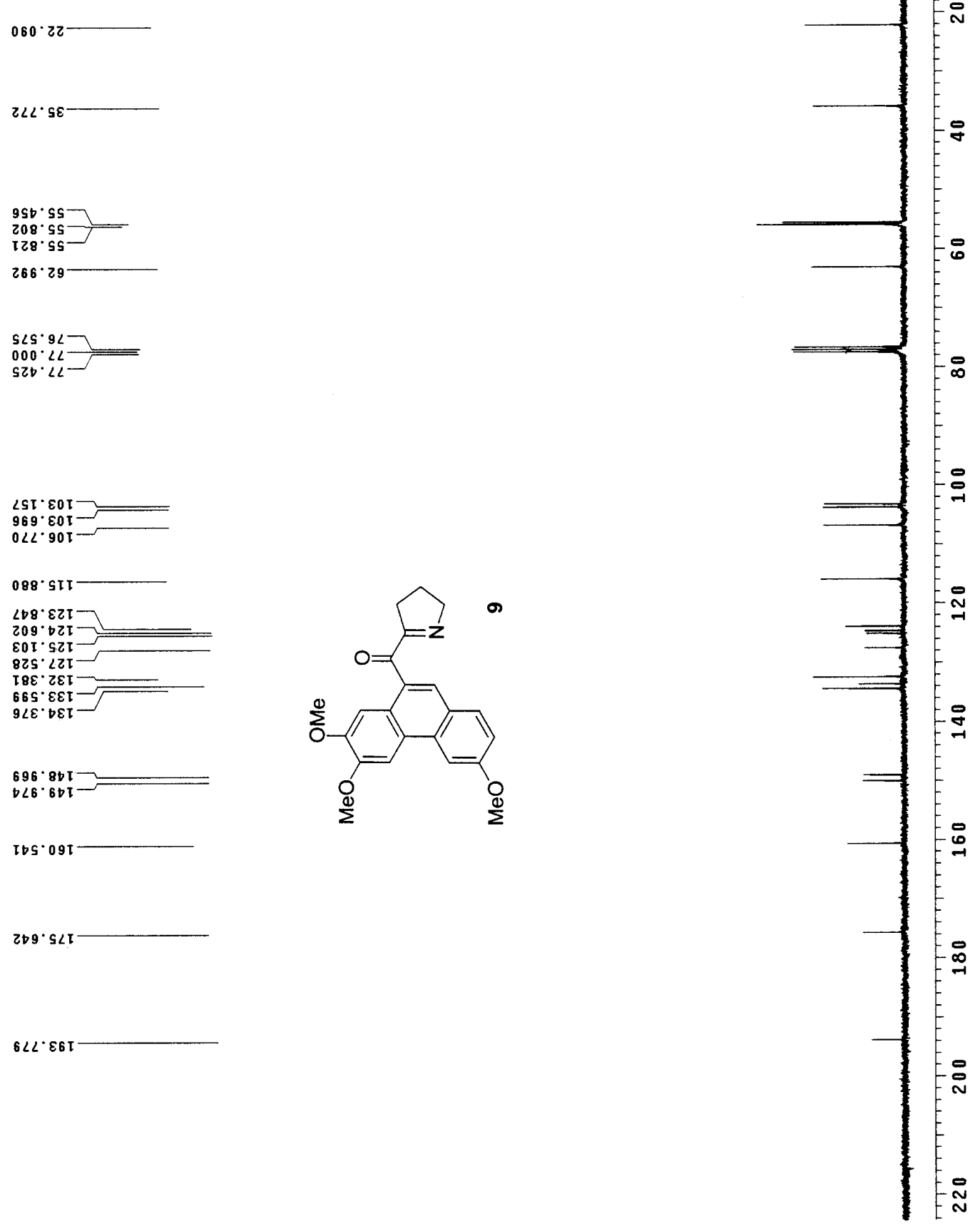




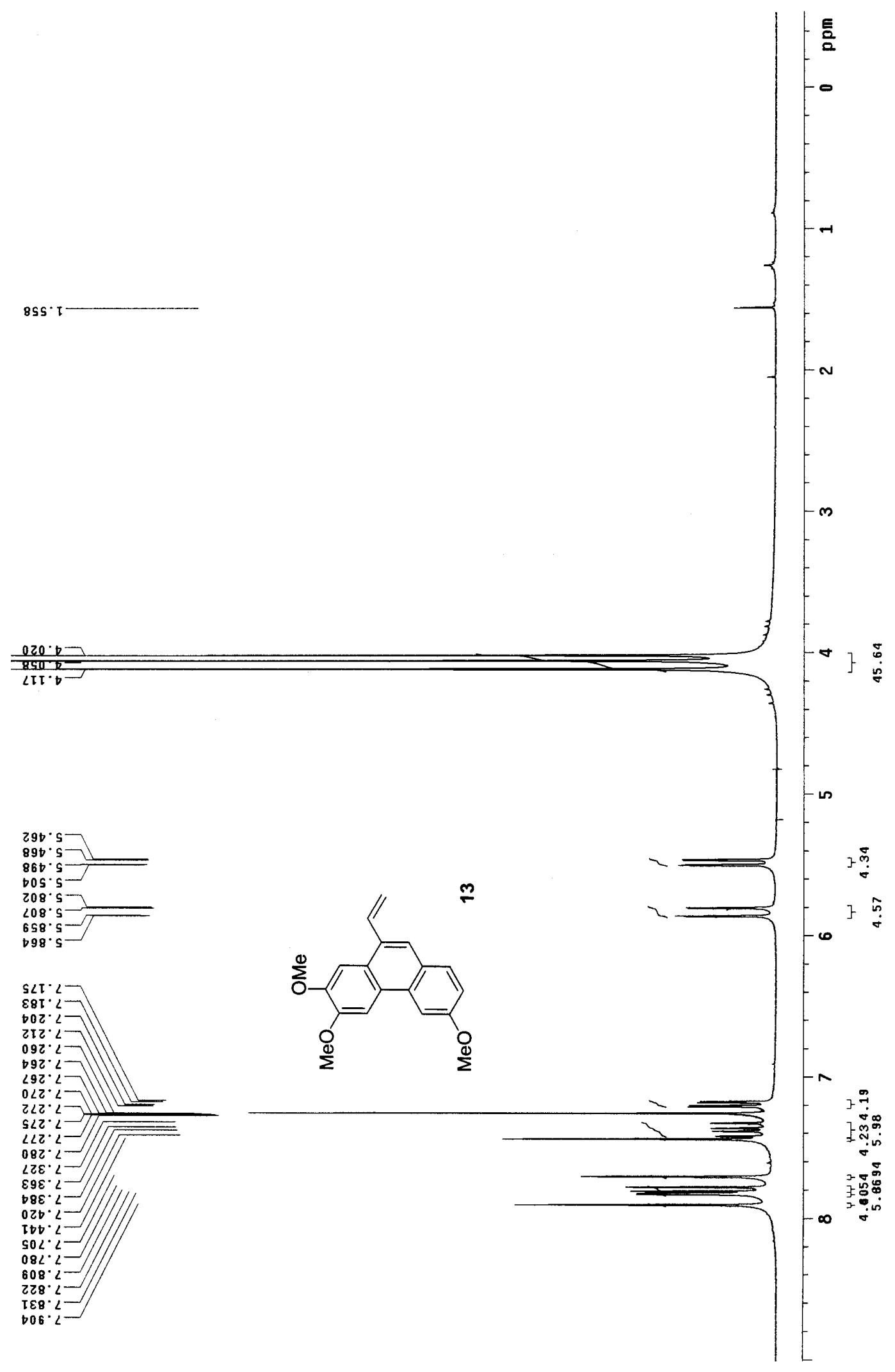


IID. SS
StL: SS
6s8. SS

$6 \angle 5 \cdot 9 L$

$000 \circ 21 \square$

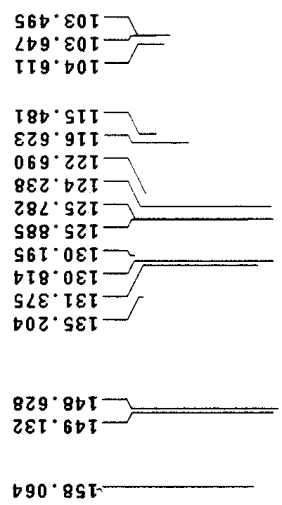

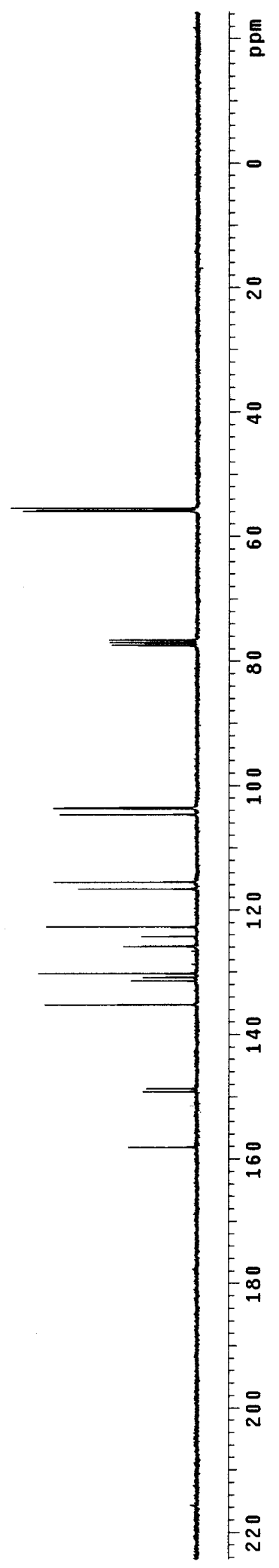



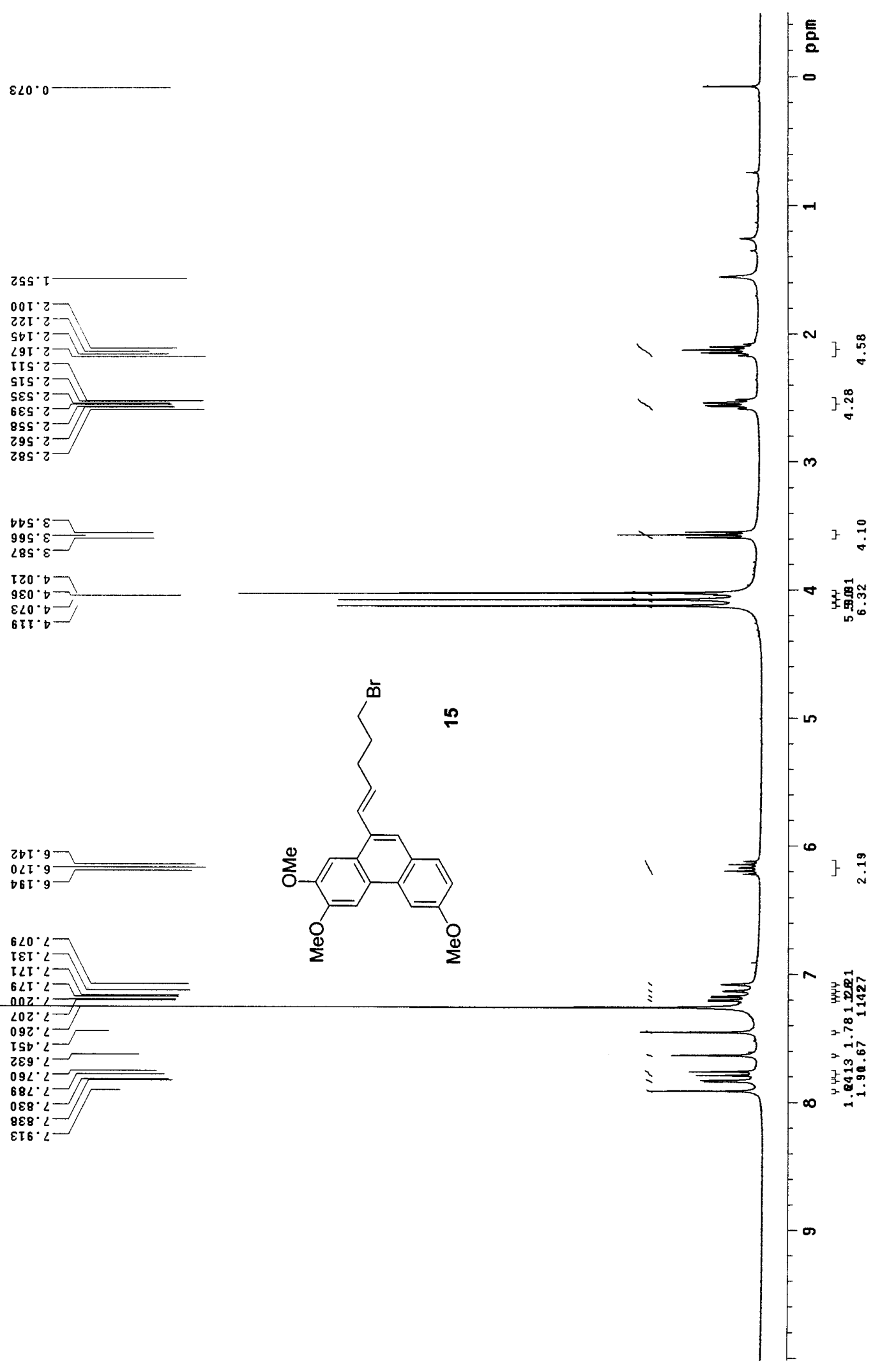
$6 \mathrm{~s} \cdot \tau \varepsilon-$

$882^{\circ} \mathrm{T} \varepsilon$

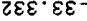

066.55

$t \varepsilon 6^{\circ} \mathrm{SS}$

$6 \angle S^{\circ} 9 \angle$ 000
$S Z 0^{\circ} L L \square$

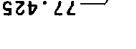

$\varepsilon \angle 9 \cdot \varepsilon 0 I-$

$662.801-$

sto.

$81 \varepsilon^{\circ} \circ 2 \mathrm{LI} \longrightarrow$

818 म2I

$202.621 \longrightarrow$

$899 \cdot 0 \varepsilon 1$

90I. IEI

$90 I \cdot I E I-$

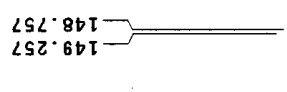

DI0.8SI

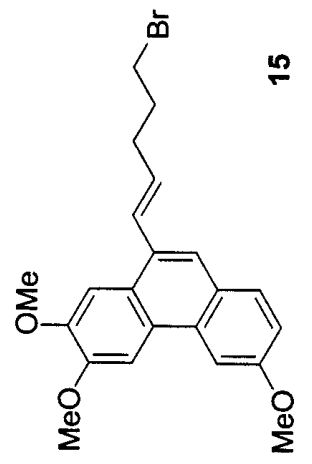

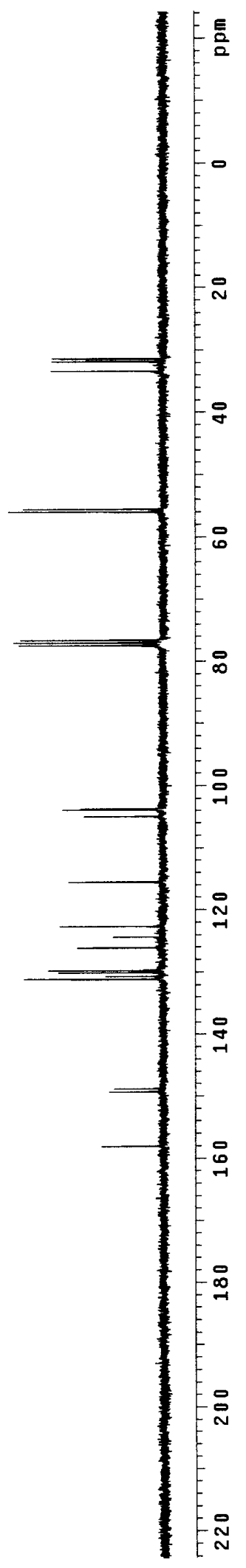



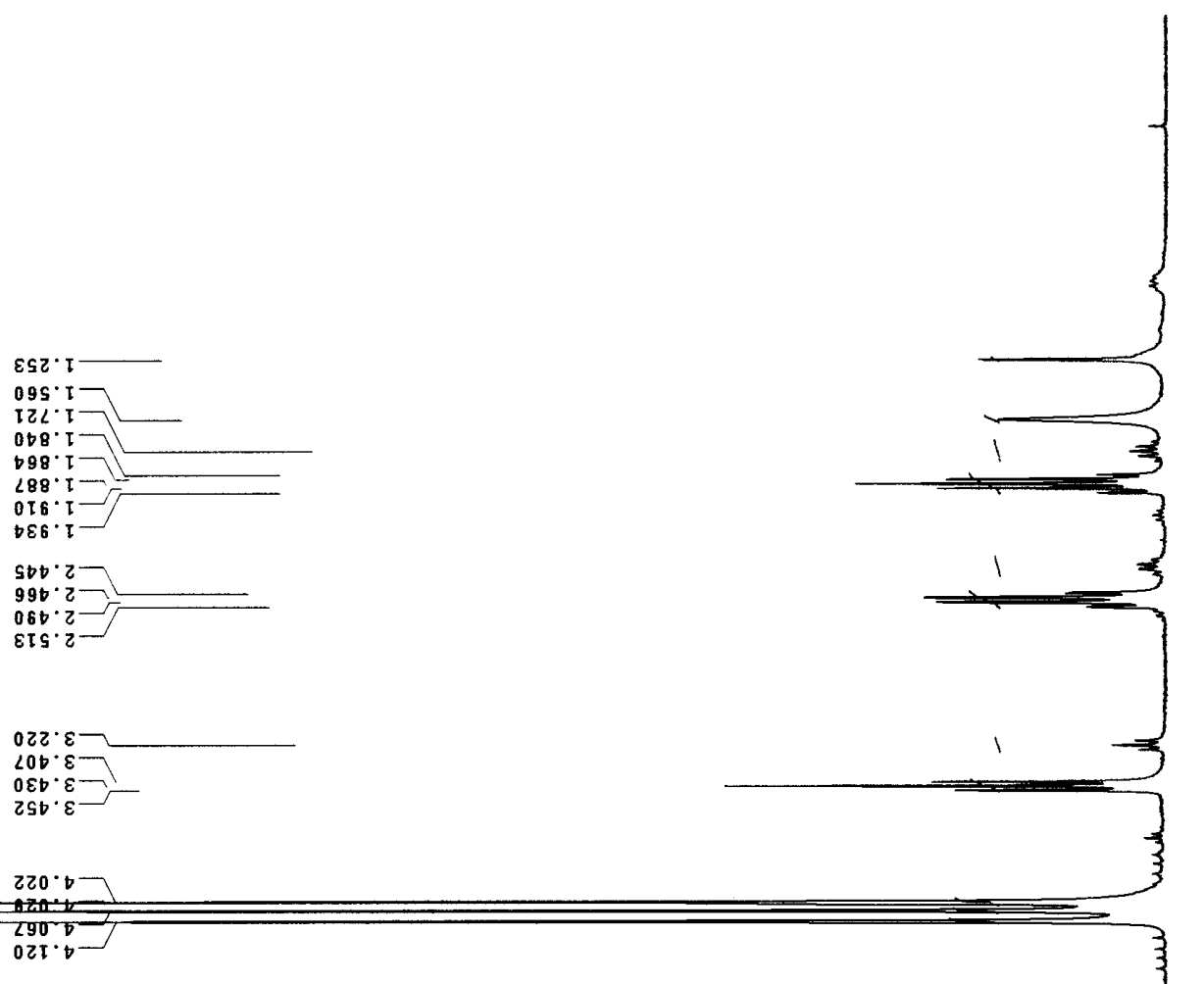

틈

-

$-$

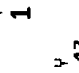

ᄀ $\stackrel{0}{?}$

อั

$-\infty$

गे

$-m$

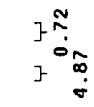

$\angle 00 \cdot \varepsilon$

${ }_{2 s b} \varepsilon^{2} \cdot \varepsilon=-$

$220^{\circ} 0$

2900

$021 \cdot 0$
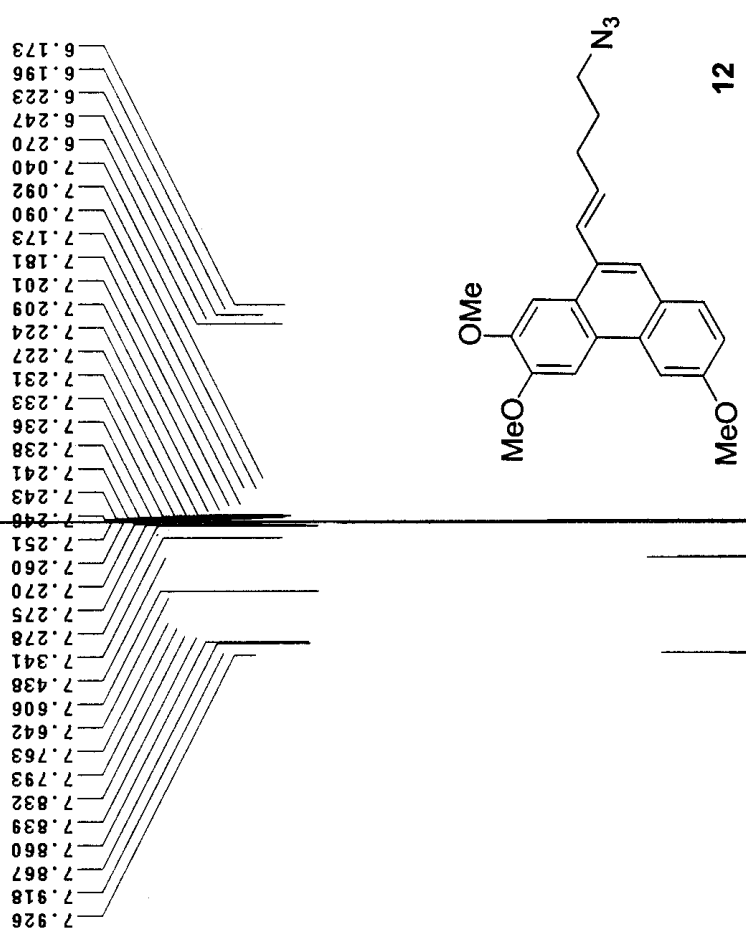

พ

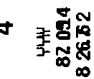

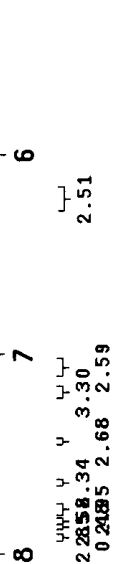



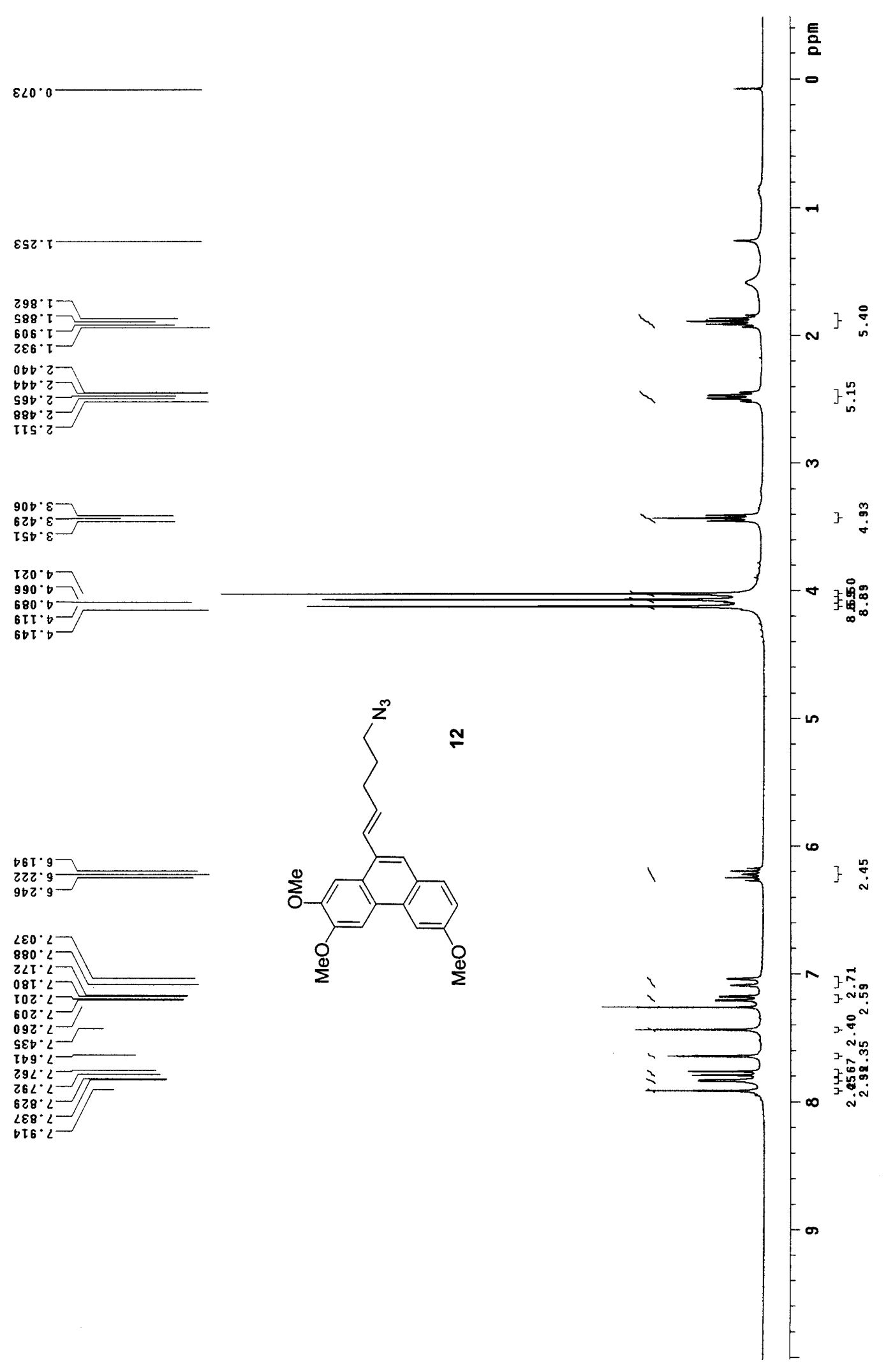

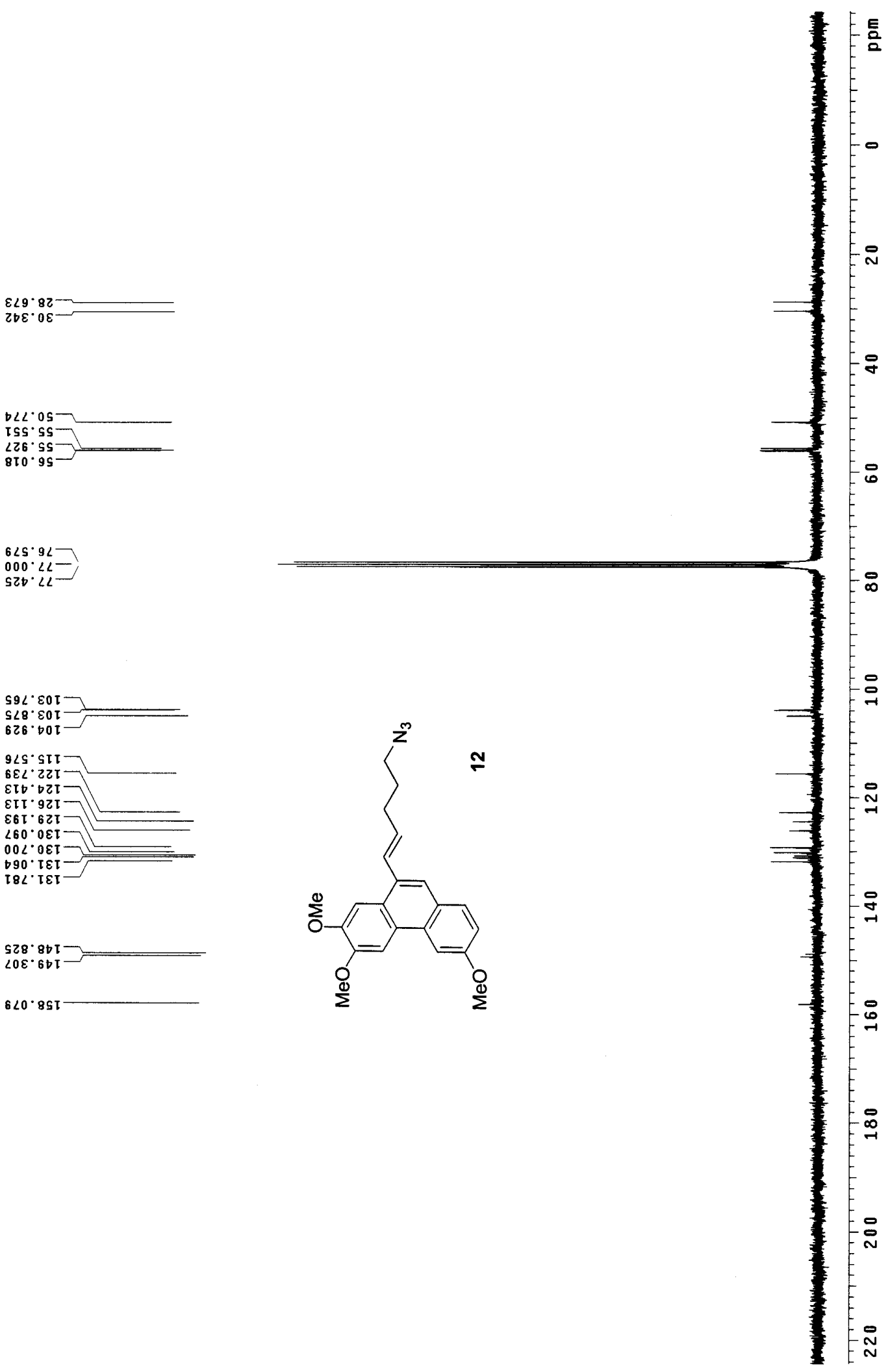


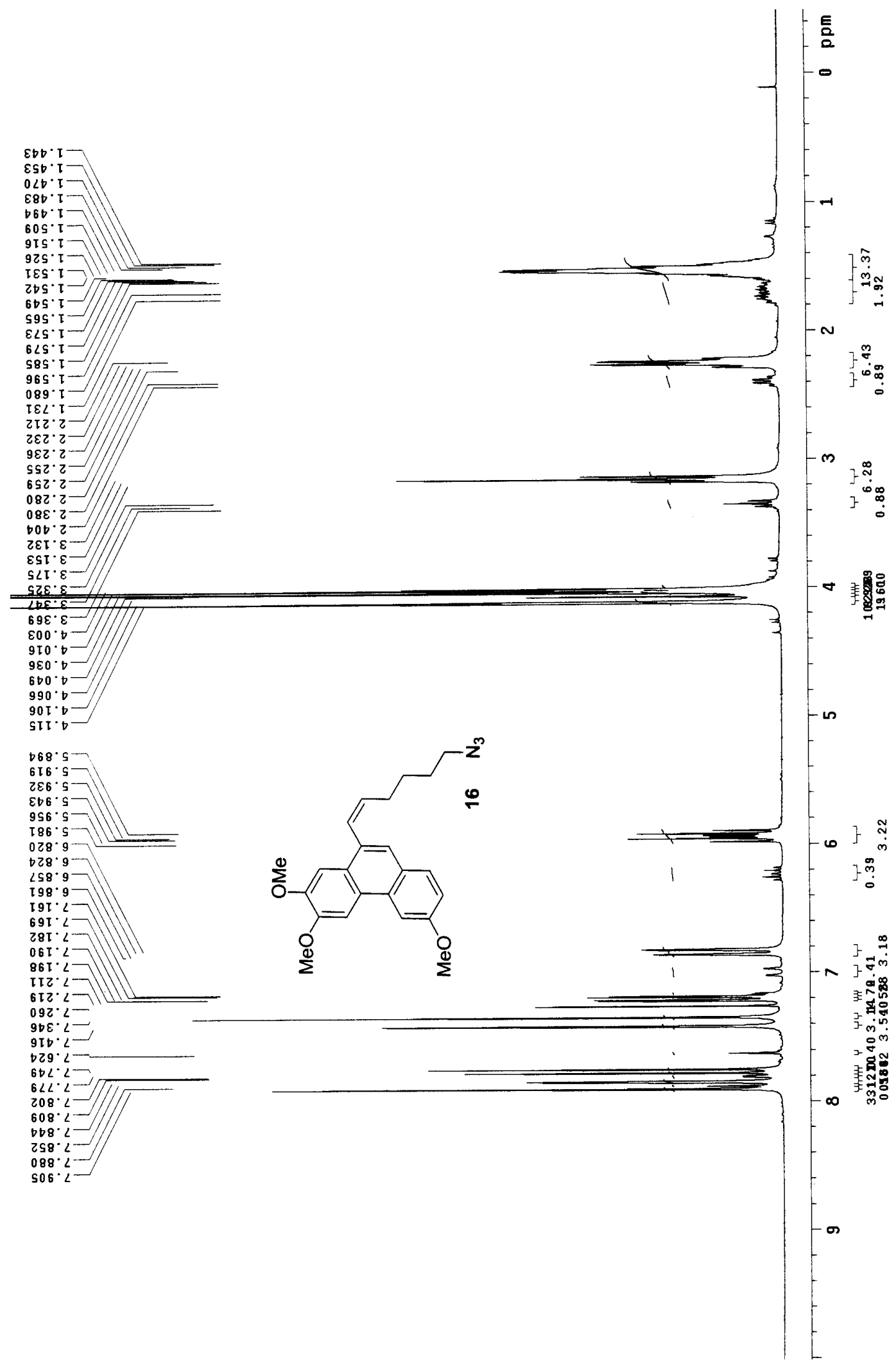



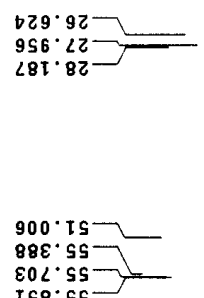

IS8. $\mathrm{ss}$

$\mathrm{S} \angle \mathrm{S}^{\circ} \mathbf{9 L}$

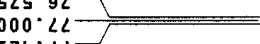

$\tau 2 t \cdot L L$
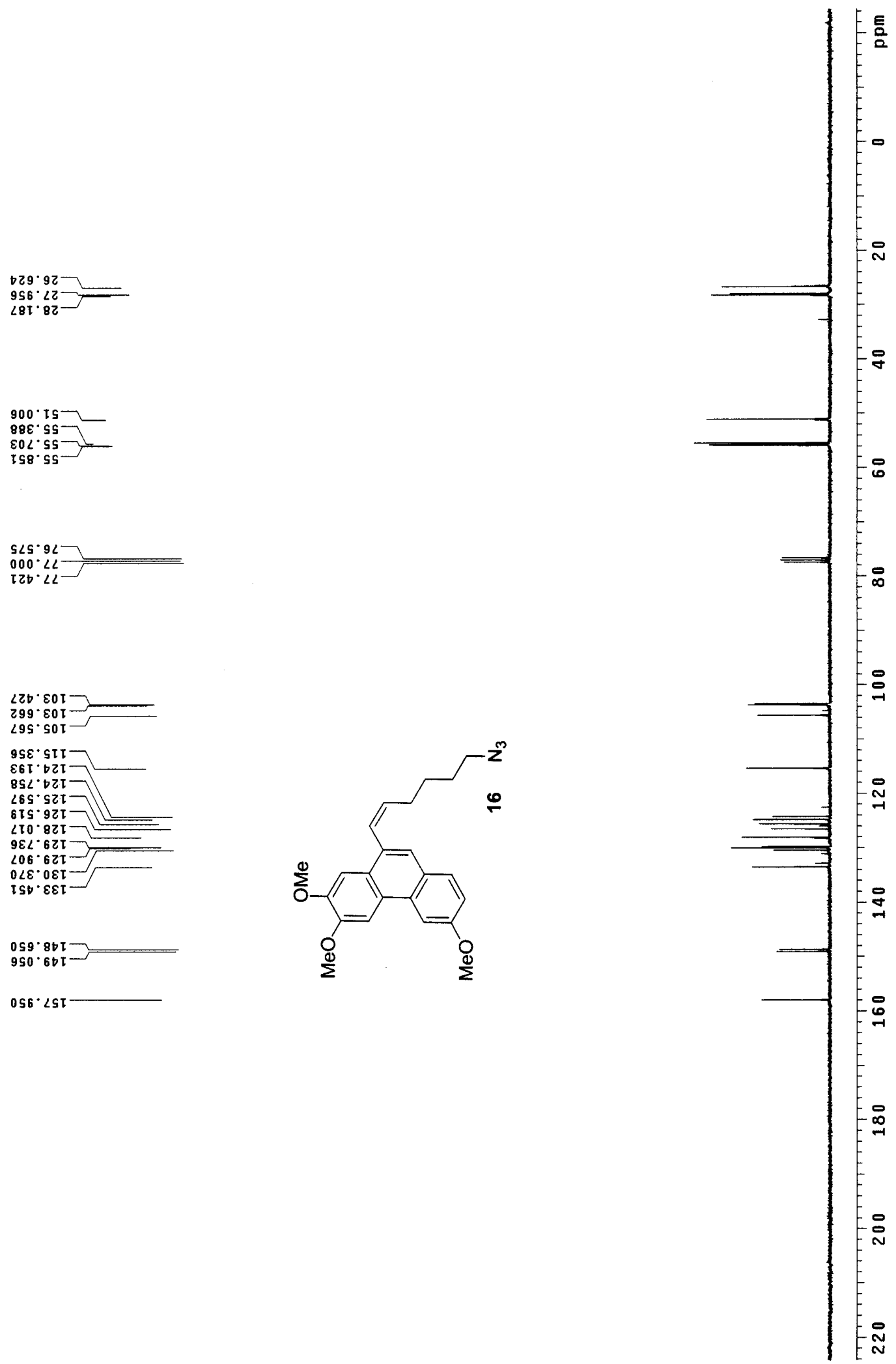

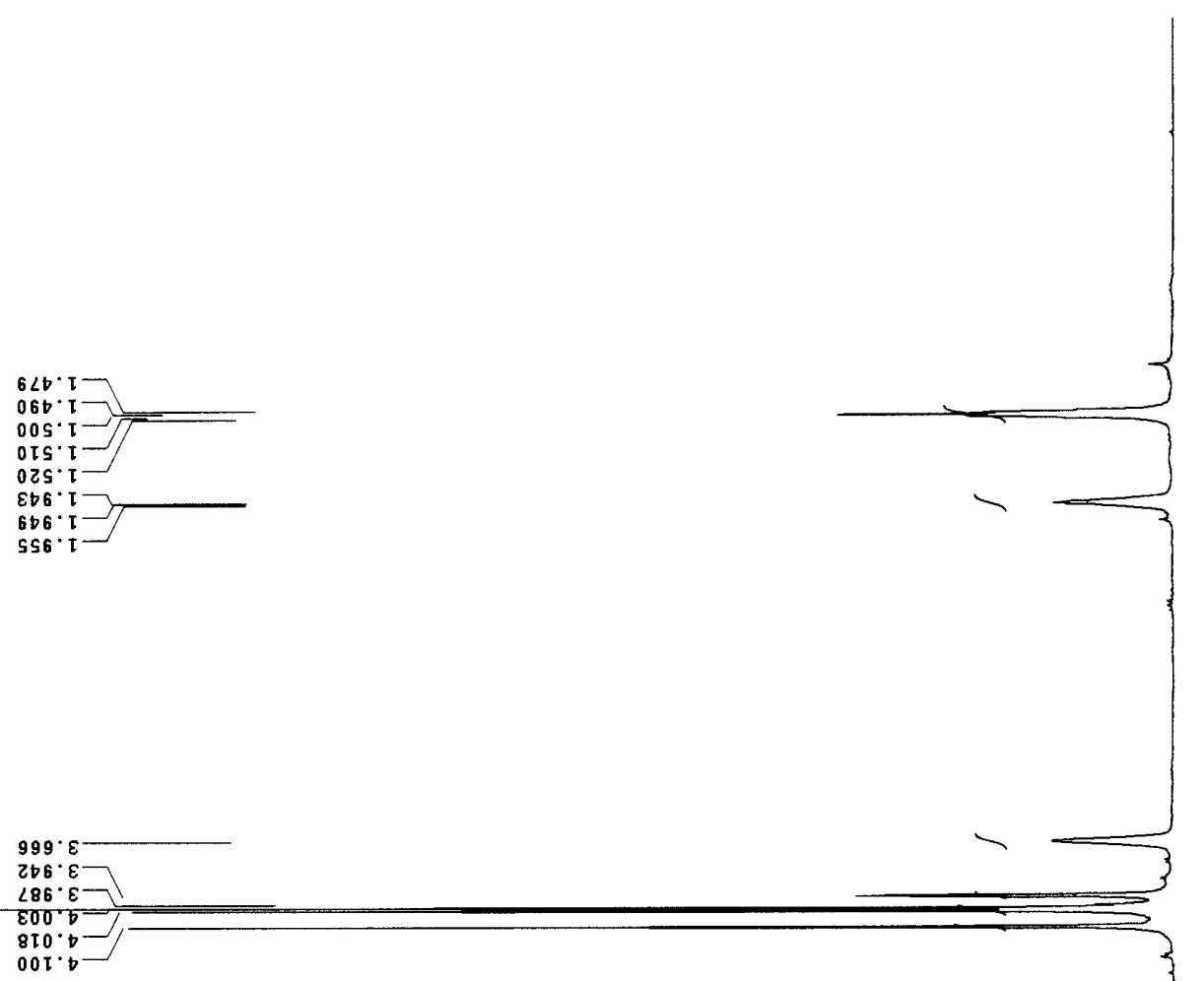

틈

$999^{\circ} \varepsilon$

$266^{\circ} \varepsilon$
$\angle 86^{\circ} \varepsilon$

$800.5=$

810.0

$00 \mathrm{I}$.

โ9เ $<7$

$691 \cdot 27$

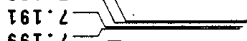

$65 \mathrm{I} \cdot 2$

$092 \cdot 2=$

SI $L \cdot 2$

s2L.

पSL:L

$828 \cdot 2-$

$988^{\circ}-$

$168^{\circ}:$

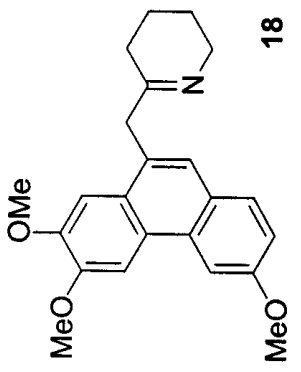

$m$

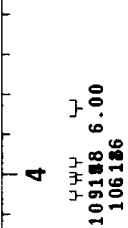

年

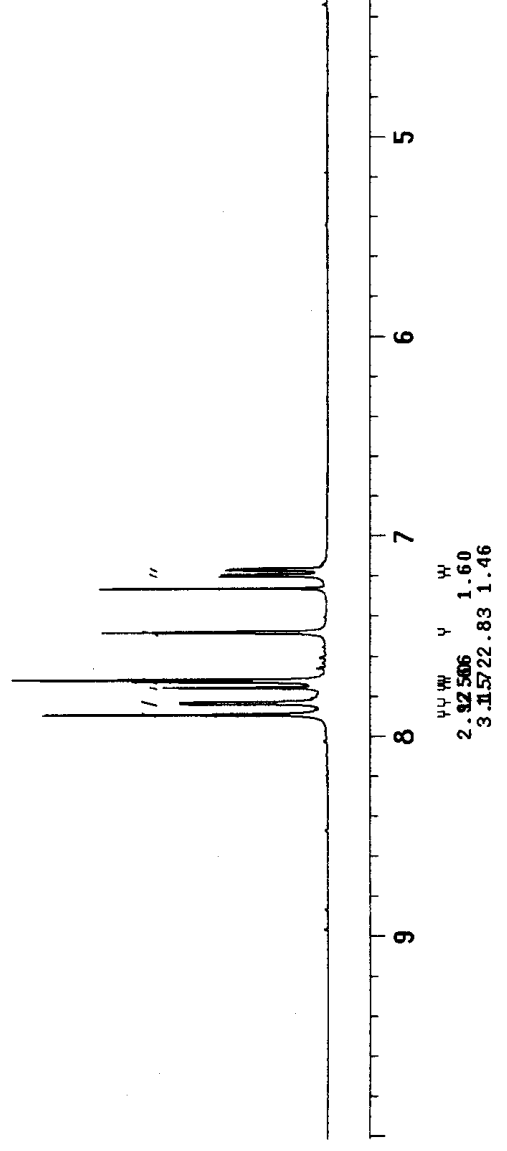



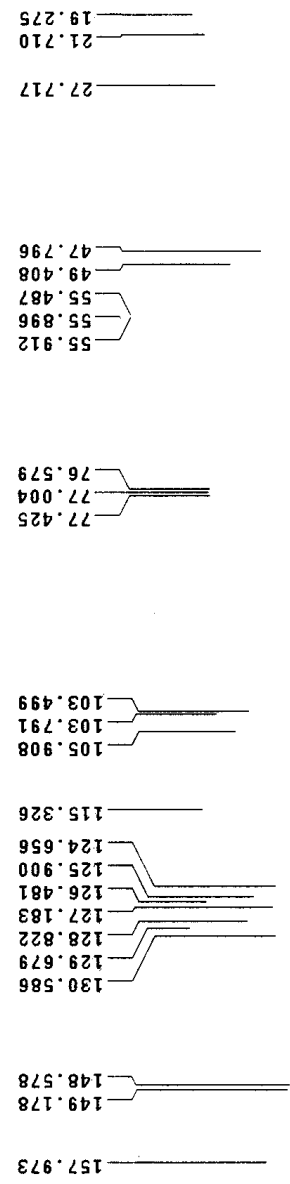

$\angle 90^{\circ} \tau \angle I$

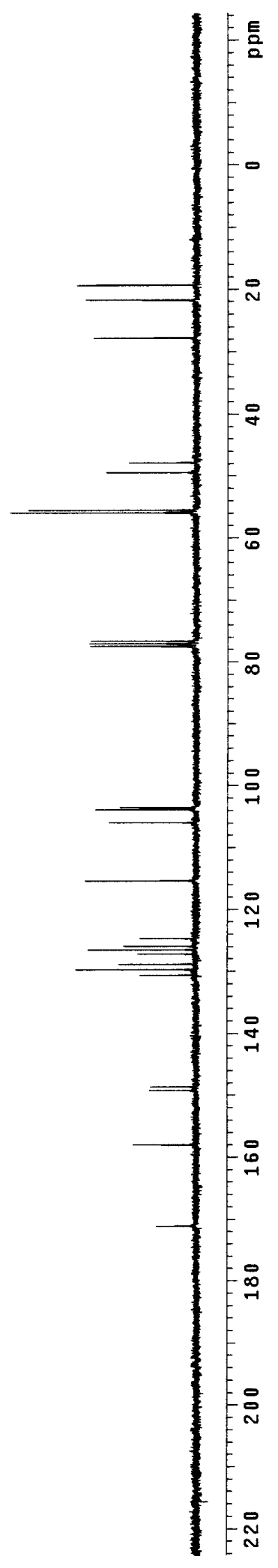

证

을

$-7$

$-8$

잉

$\operatorname{Nan}^{2}$

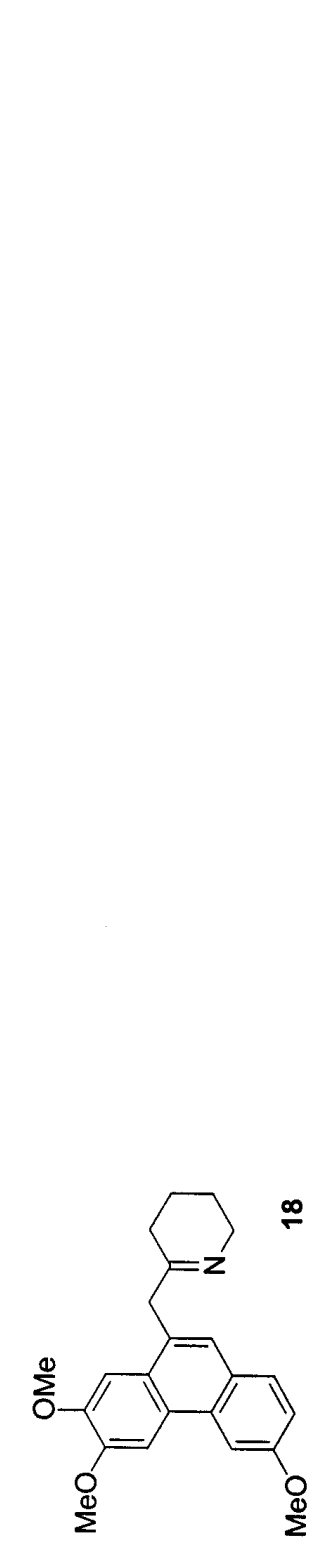




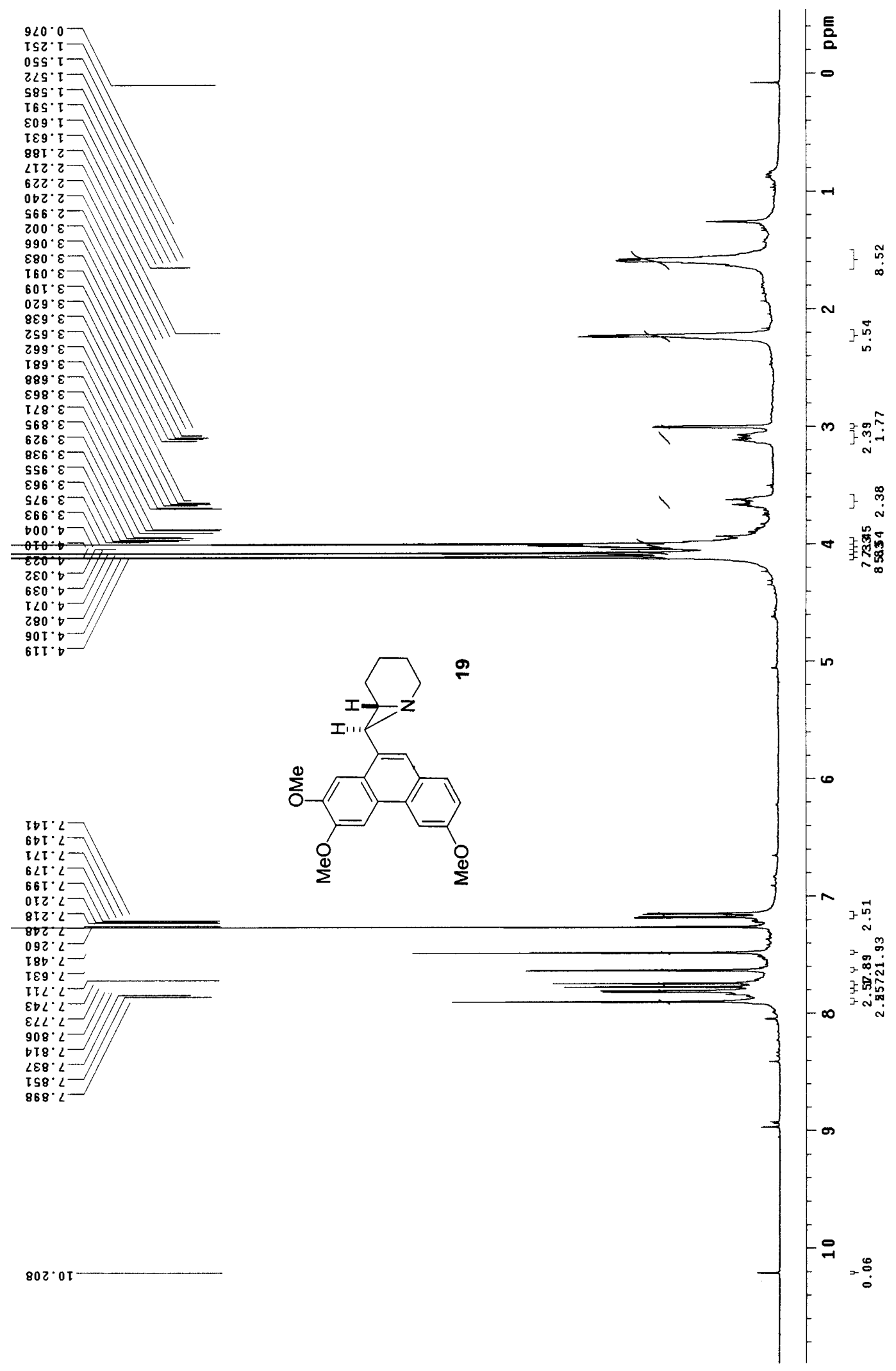


$\angle I b \cdot 8 \tau$

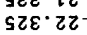

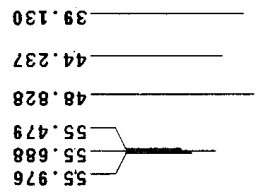

$5 \angle 5 \cdot 9<$

000.22

I $20 \cdot \angle L$

$0 b 8 \cdot \varepsilon 0 . \tau$

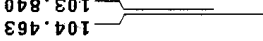

$2 \angle Z \cdot s i t$

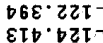

$6 Z 0.921$

$02 L^{\circ} 92 \mathrm{I}$

$\angle 60^{\circ} 0 \varepsilon \mathrm{I}$

$\tau \varepsilon \varepsilon \cdot 0 \varepsilon \mathfrak{I}$

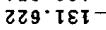

$\varepsilon \nabla 9^{\circ} 8 t \tau$

$60 \tau \cdot 6 b \tau$

$\varepsilon 68^{\circ} \mathrm{LST}$

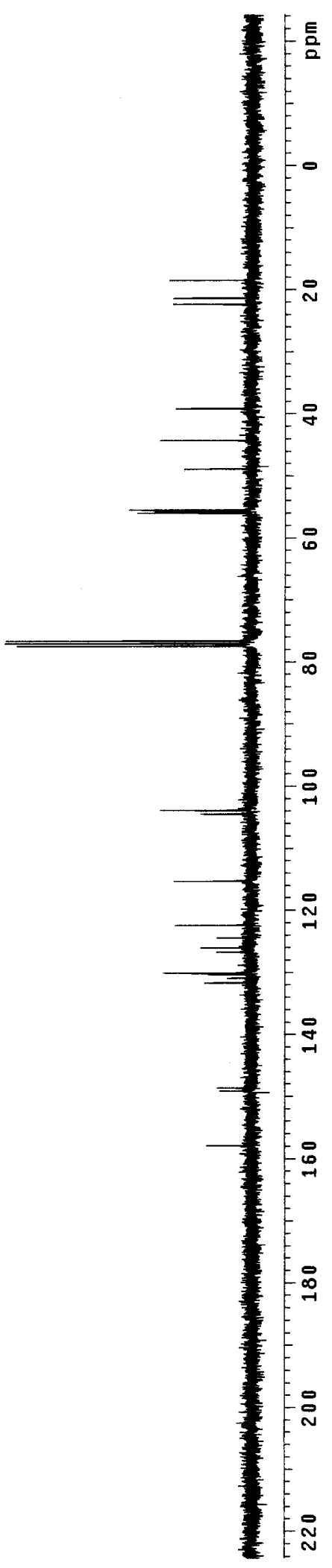

